\title{
Chewing gum for intestinal function recovery after caesarean section: a systematic review and meta-analysis
}

\author{
Zunjia Wen ${ }^{1,2+}$, Meifen Shen ${ }^{1,2^{*}{ }^{+}}$, Chao $\mathrm{Wu}^{1+}$, Jianping Ding ${ }^{2}$ and Binbin $\mathrm{Mei}^{2}$
}

\begin{abstract}
Background: Gum chewing has been reported to enhance the intestinal function recovery after caesarean section, current perspectives and practice guidelines vary widely on the use of gum chewing, more studies on the role of gum chewing after caesarean section are needed.

Methods: We performed a comprehensive, systematic meta-analysis of randomized controlled trials (RCTs) on the efficacy of gum chewing after caesarean section. Studies were identified by searching EMBASE et al database (until June 30,2016$)$. Summary odd ratios or weighted mean differences with $95 \%$ confidence intervals were calculated for each outcome with fixed- or random-effects model.

Results: Ten RCTs with a total of 1659 women were included in our meta-analysis. Gum chewing provided significant benefits in reducing the time to first passage of flatus, first defecation, first bowel sound, first bowel movement and the length of hospital stay, but not in the time to first feeling of hunger.

Conclusions: Gun chewing hastens the intestinal function recovery after caesarean section and offers a safe and inexpensive option. High-quality and larger-scale RCTs are still warranted to clarify the role of gum chewing in intestinal function recovery after caesarean section.
\end{abstract}

Keywords: Caesarean, Gum, Intestinal function, Meta-Analysis, Recovery, Review

\section{Background}

With the development of medical care and policy supports, the caesarean delivery, a most commonly seen operation, has increased worldwide over the past decades [1]. However, it may lead to many complications such as postoperative ileus with a mean incidence of $10-15 \%[2,3]$, result in longer hospital stay, increased postoperative morbidity and excessive medical costs [4].

Promoting intestinal function recovery after caesarean section is on the top of research agenda of healthcare providers. Traditionally, physicians forbid oral feeding with concern to the risks of intestinal fistula, re-bleeding and aspiration mistakenly [5], but recent studies have supported that early postoperative feeding can stimulate bowel motility and shorten hospital stay $[6,7]$, however,

\footnotetext{
*Correspondence: smf8165@126.com

${ }^{\dagger}$ Equal contributors

${ }^{1}$ The First Affiliated Hospital of Soochow University, No. 188 Shizi Street, Gusu district, Su Zhou, Jiangsu province, China

${ }^{2}$ Nursing School of Soochow University, Su Zhou, China
}

patients may not tolerate the early feeding regimen [8]. Several methods have been proposed to accelerate the return of gastrointestinal motility after caesarean section, including early oral hydration, ambulation and gum chewing.

Gum chewing as a kind of sham feeding was introduced in hope that it may hasten the intestinal function recovery in recent years, by means of stimulating the cephalic vagal reflex, the hormones secretion may increase [9]. The efficacy of gum chewing have been fully elucidated in the intestinal function recovery in patients after colorectal surgery and it can provide various benefits $[10,11]$, yet the efficacy of chewing gum after caesarean section remain inconsistent. Several previous meta-analysis [12-17] including randomized controlled trials (RCTs) on the gum using after caesarean have been conducted, but the included data are rather limited. Up to date, no guideline has officially support the use of chewing gum for intestinal 
function recovery in obstetrics and gynaecology, more evidences of higher quality on this issue are warranted.

Given the recently emerging evidence on the role of gum chewing after caesarean section, we performed this systematic review and meta-analysis of RCTs with the following objectives: (1) to review and sum up the current evidence on the influence of gum chewing in intestinal function after caesarean section; (2) to compare the efficacy of chewing gum and standard care in intestinal function recovery after caesarean section; and (3) to analyze and conclude the appropriateness of gum chewing in patients after caesarean section.

\section{Methods}

\section{Search strategy}

We tried to plan, perform and report this meta-analysis in comply with PRISMA guideline [18]. Related articles either published in English or Chinese were identified and selected by searching PUBMED, EMBASE, Science Direct, Cochrane Central Register of Controlled Trials, China National Knowledge Infrastructure (CNKI) and Wanfang Database (until June 30, 2016) using the following search terms including "gum-chewing", "chewing-gum", "sham-feeding", "caesarean section", "caesarean", "caesarean delivery", we combined these terms in accordance to the instructions of the database. In addition, the reference lists of the retrieved studies and pervious reviews and meta-analyses were reviewed and manually searched, and we made no attempt to identify unpublished reports.

\section{Study selection}

Study selection was made based on a first screen of identified titles or abstracts and on a second check-up of full-text articles. Studies were considered to be eligible if the criteria below were met: (1) RCT design; (2) study subjects had receiving caesarean section; (3) included the comparison groups of added gum chewing and standard nursing care post caesarean delivery; and (4) the relative outcome data (the time to first passage of flatus after operation, et al) were reported. Studies were excluded if: (1) it's not RCT design, which may produce more heterogeneity for data analysis; (2)the study subjects had not received caesarean section; (3) the relative outcome data were not accessible for data synthesis.

\section{Data extraction}

The following data information were extracted by two reviewers independently: first author, year of publication, country, patient population, participants, methods of gum chewing, other concurrent interventions, main outcomes and study results. The studies selection and data extraction were conducted by two authors independently, any disagreement was resolved by further discussion.

The outcomes we collected for data analysis included: (1) the time to first passage of flatus; (2) the time to first defecation; (3) the time to first bowel movement; (4) the length of hospital stay; (5) time to first feeling of hunger; (6) the time to first bowel sound; (7)postoperative complications such as nausea, abdominal distension, vomiting et al.

\section{Assessment of the quality of the included studies}

The Cochrane Collaboration's "risk of bias" tool was adopted for evaluating the methodological quality and risk of bias of included RCTs, seven specific domains were examined and measured in this tool: sequence generation, allocation concealment, blinding of participants and personnel, blinding of outcome assessment, incomplete outcome data, selective outcome reporting, and "other" issues. Every domain could be classified as "low risk of bias", "high risk of bias" or "unclear risk of bias" under the guidance of judgment criteria (Cochrane Handbook for Systematic Reviews of Intervention. Part 2: 8.5).

\section{Data synthesis and analysis}

All the extracted data were processed in a freeware program Review Manager (RevMan) Version 5.3. Binary outcomes (i.e., VAP and mortality) were presented as Mantel-Haenszel style odd ratios (ORs) with 95\% confidence intervals, and continuous outcomes were reported as inverse variance weighted mean differences (WMDs). A fixed-effect model was adopted in cases of homogeneity ( $p$ value of $\chi 2$ test $>0.10$ and $\mathrm{I}^{2}<50 \%$ ), while a random-effects model was used in cases of obvious heterogeneity ( $p$ value of $\chi 2$ test $>0.10$ and $\mathrm{I}^{2}>50 \%$ ). Publication bias was evaluated by the demonstration of funnel plots, and asymmetry was assessed by means of the Egger regression test ( $p$-value $<0.1$ was considered to be significant of funnel plot asymmetry).

\section{Results}

\section{Literature search}

A sum of 528 relevant publications were yielded by the comprehensive search, and the first screen of titles and abstracts excluded 473 papers, and 55 potentially related studies were included for further full-text review, participants and interventions not meeting the including criteria were the main concern for further consideration, eventually, 10 RCTs [19-28] were included for data analyses (Fig. 1).

\section{Study characteristics}

The basic characteristics of 10 included studies are shown in Table 1 . In brief, a total of 1659 patients were involved, specifically with 835 patients for gum chewing 


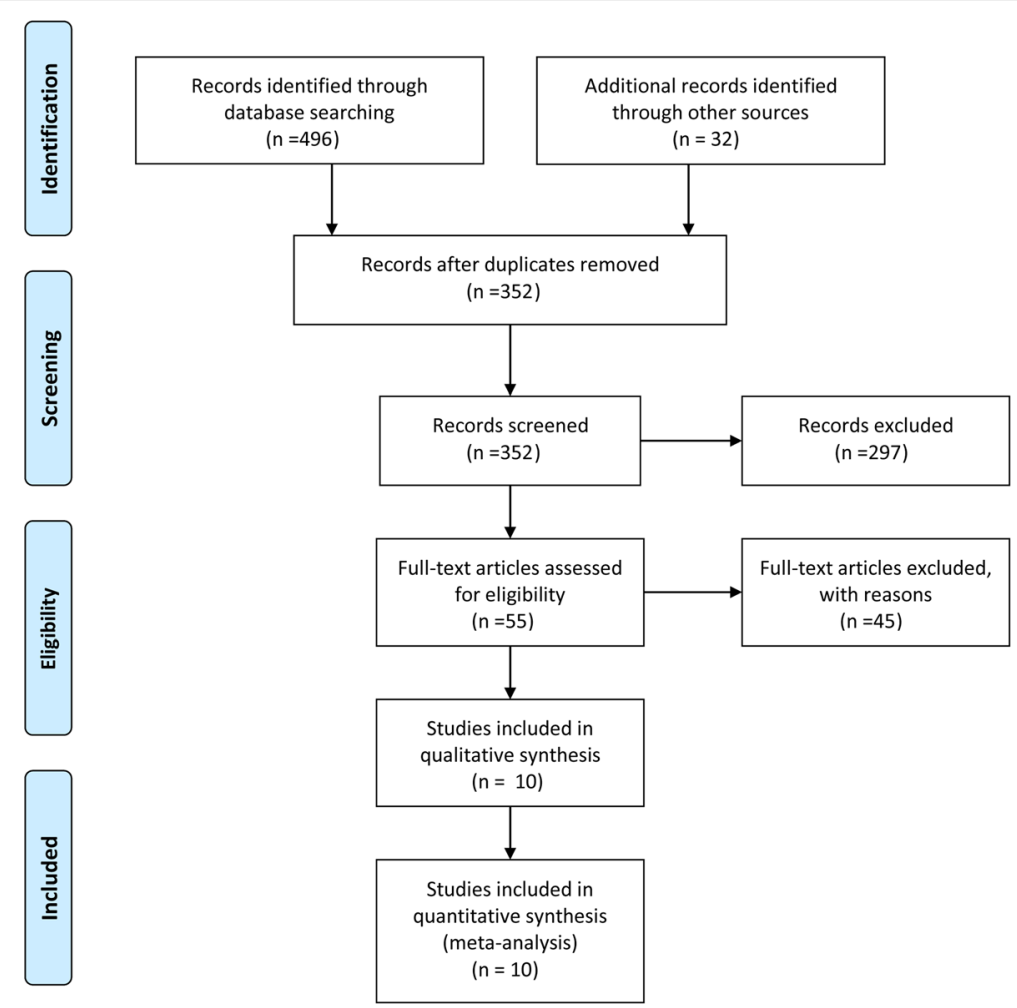

Fig. 1 Flow diagram of study selection

intervention, and 824 for standard postoperative care, the countries of included studies ranged from Egypt, Nigeria, Thailand, Turkey, Iran, Philippines to China, and four RCTs were conducted in China, the numbers of included participants among studies differed from 50 to 386. It's noteworthy that one study was reported as conference paper, although the structure and data were relatively complete, the details on how study conduct remain unclear, we attempted to contact the original authors for more information, yet no response was achieved, therefore, we still included this study for data analysis, but we put more cautions on the quality of study details. All studies have reported no statistical significant differences in the baseline characteristics between intervention and control groups. Meanwhile, some differences among studies were found in the gum chewing interventions, including the timing of initiation and frequency of gum chewing, one study [24] also compared the efficacy of xylitol-containing and xylitol-free gum chewing with standard postoperative care protocol, in our study we only extracted the data in xylitol-free gum chewing and control groups. Interestingly, all the results favored the usage of gum chewing after caesarean sections.

\section{Methodological quality and risk of bias}

Figure 2a illustrates all of the bias classifications in a way of cross-tabulation for the included RCTs, and the summary of qualitative methodological quality abiding by the bias classification ("Low risk", "High risk", "Unclear risk") is presented in Fig. 2b. Briefly, all included RCTs mentioned that the randomization was adopted in their studies, yet three RCTs [25, 26, 28] made no reports on the methods for random sequence generation, only two studies reported the strategies to achieve allocation concealment and then were marked as low bias of risk. Considering that it's hard to blind patients and care providers on the gum chewing intervention, most RCTs adopted single-blind or no blind study design instead of a double-blind study design, all RCTs made no blind study design on participants and personnel then were classified as high risks of bias, and only two $[19,27]$ adopted the blind design on outcome assessment. Except for the details lack of Zaroma' study [28],all the other RCTs have provided the necessary data and outcomes for selective reporting and other bias evaluations.

\section{Main analysis}

The WMDs for each study on the time to first passage of flatus, the time to first defecation, the time to first bowel sound, the length of hospital stay, the time to first bowel movement, time to first feeling of hunger are shown in Fig. 3. 
Table 1 The characteristics of included studies

\begin{tabular}{|c|c|c|c|c|c|}
\hline Author(year) & Country & $\begin{array}{l}\text { Numbers of } \\
\text { participants(GC/NGC) }\end{array}$ & Gum intervention & Main outcomes & Results \\
\hline $\begin{array}{l}\text { Abd-El-Maeboud } \\
2009 \text { [19] }\end{array}$ & Egypt & $200(93 / 107)$ & $\begin{array}{l}\text { Started from } 2 \mathrm{~h} \text { after surgery, lasted } \\
\text { for } 15 \text { min every } 2 \mathrm{~h} \text { in the daytime } \\
\text { but not in the night, until the } \\
\text { passage of flatus }\end{array}$ & (1),(2, (4),(5) & $\begin{array}{l}\text { Gum chewing after CS is safe, well } \\
\text { tolerated, and associated with rapid } \\
\text { resumption of intestinal motility and } \\
\text { shorter hospital stay }\end{array}$ \\
\hline Ajuzieogu 2014 [20] & Nigeria & 180(90/90) & $\begin{array}{l}\text { Started from the first day after } \\
\text { operation for } 5 \text { consecutive days, } 3 \\
\text { times daily, } 30 \text { min for every time. }\end{array}$ & (1),(2, (4), (5) & $\begin{array}{l}\text { Gum chewing has a beneficial effect } \\
\text { on early return of bowel function } \\
\text { following cesarean section and should } \\
\text { be included in the postoperative } \\
\text { management protocol }\end{array}$ \\
\hline Jakkaew 2013 [21] & Thailand & $50(25 / 25)$ & $\begin{array}{l}\text { Started since the regain of } \\
\text { consciousness after operation, } 4 \\
\text { times a day (morning, noon, evening, } \\
\text { and before bed time), } 30 \text { min for } \\
\text { every time, until the first passage } \\
\text { of flatus }\end{array}$ & (1),(5,(6, (7) & $\begin{array}{l}\text { Gum chewing is associated with } \\
\text { faster recovery of bowel function } \\
\text { following cesarean section }\end{array}$ \\
\hline Kafali 2010 [22] & Turkey & $150(74 / 76)$ & $\begin{array}{l}\text { Started from } 2 \mathrm{~h} \text { after surgery, } 3 \\
\text { times daily in the morning, } \\
\text { afternoon, and evening, each } \\
\text { episode of gum chewing lasted } 1 \mathrm{~h} \\
\text { except the initial one which lasted } \\
15 \text { min }\end{array}$ & (1),(4),(5) & $\begin{array}{l}\text { Gum chewing provides a simple } \\
\text { method for early recovery of bowel } \\
\text { function after cesarean section }\end{array}$ \\
\hline Ledari 2012 [23] & Iran & $100(50 / 50)$ & $\begin{array}{l}\text { Chewing gum for at least } 1 \mathrm{~h} \text {, three } \\
\text { times daily from } 6 \mathrm{~h} \text { after surgery } \\
\text { (after recovery from anesthesia) } \\
\text { until being discharged }\end{array}$ & (1),4,6) & $\begin{array}{l}\text { chewing is acceptable and inexpensive } \\
\text { physiologic method for decreasing the } \\
\text { time to the passage of flatus, bowel } \\
\text { movements, and feeling of hunger in } \\
\text { patients undergoing cesarean section }\end{array}$ \\
\hline Lee 2016 [24] & China & $120(40 / 40 / 40)$ & $\begin{array}{l}\text { Chewing xylitol-free or xylitol- } \\
\text { containing gum started from } 2 \mathrm{~h} \\
\text { after surgery, repeated every } 2 \mathrm{~h} \\
\text { between } 9 \text { a.m. and } 8 \text { p.m, } 15 \text { min }\end{array}$ & (1),(2, (4) & $\begin{array}{l}\text { After cesarean section, chewing gum } \\
\text { increased participants' return of bowel } \\
\text { activity and xylitol-containing gum } \\
\text { may be superior to xylitol-free gum }\end{array}$ \\
\hline
\end{tabular}

Liang 2007 [25] China 120(60/60)

Luo 2010 [26] China 300(150/150)

Shang 2010 [27] China 386(195/191)

Zamora 2012 [28] Philippines 53(18/35) for every time, until the first passage of flatus

Started after recovery from anesthesia, repeated every $2 \mathrm{~h}$ for $15 \mathrm{~min}$ every time

Started from $2 \mathrm{~h}$ after surgery, 4 times daily in the morning, afternoon, and evening, each episode of gum chewing lasted 10-15min

Chew gum for at least half an hour, three times per day after returning to the ward

Gum chewing involved chewing two pellets of sugarless gum at $12 \mathrm{~h}$ post operation for $15 \mathrm{~min}$ then advanced to sips of clear liquids at $16 \mathrm{~h}$ post operation
Chewing gum can promote gastrointestinal motility recovery after cesarean section, and the method is simple, convenient, safe

(1),(2, (4),(7),(9) Chewing gum can promote the recovery of gastrointestinal function after cesarean section

(1),(2),(4),(5),(1) Gum chewing is an inexpensive, convenient, and physiological method in enhancing the recovery of bowel function

Postoperative gum chewing stimulates the earlier return of bowel motility after cesarean delivery

Notes: (1), the time to first passage of flatus; (2), the time to first defecation; (3), the time to first bowel movement; (4), the time to first bowel sound; (5), the length of hospital stay; (6, time to first feeling of hunger; (7), abdominal distension; (8), vomiting; (9, post operation incision pain; (10, postoperative ileus(POI)

The time to first passage of flatus All included studies have reported the time to the first passage of flatus, The summary WMDs on the time to first passage of flatus (hours) was -5.91(95\% CI: -7.08- -4.73), with evidence of heterogeneity $\left(P=0.005, \mathrm{I}^{2}=61 \%\right)$ (Fig. 3a).

The time to first defecation Seven studies $[19,20$, 23-27] have reported the time to the first passage of flatus, The summary WMDs on the time to first defecation (hours) was -7.91(95\% CI: $-11.14--4.67)$, with evidence of heterogeneity $\left(P<0.001, \mathrm{I}^{2}=93 \%\right)$ (Fig. 3b).

The time to first bowel sound Six studies [19, 20, 22$24,26]$ have reported the time to first bowel sound, The summary WMDs on the time to first bowel sound 


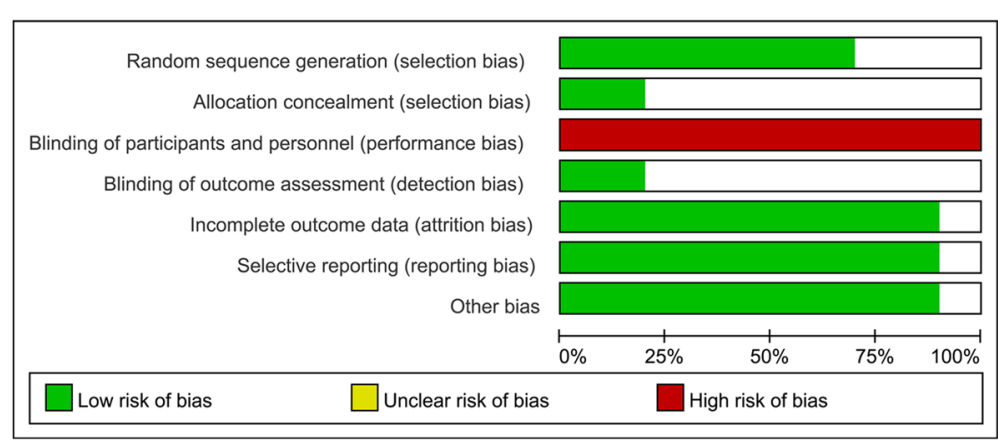

A Risk of bias graph

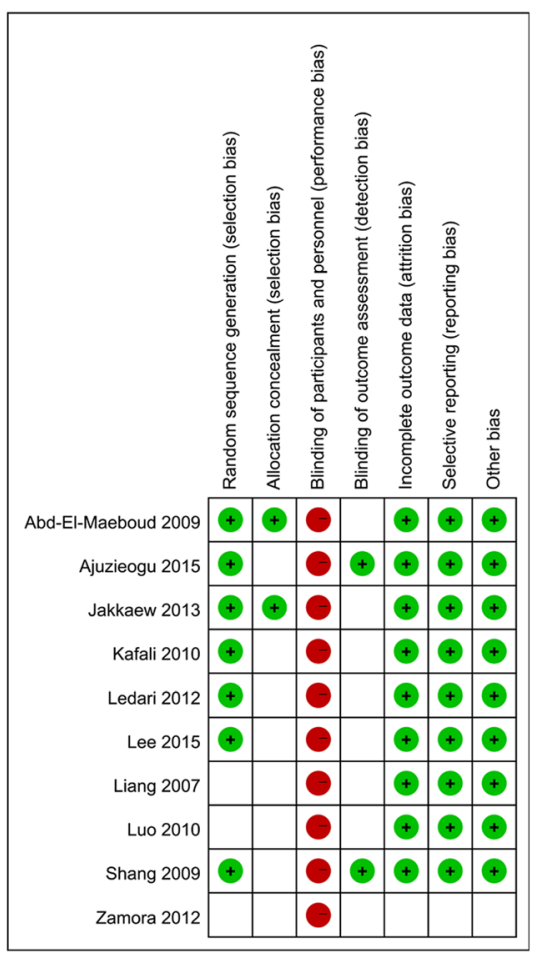

B Risk of bias summary

Fig. 2 Methodological quality and risk of bias of the included studies

(hours) was -4.19(95\% CI: -6.41- -1.97), with evidence of heterogeneity $\left(P<0.001, \mathrm{I}^{2}=97 \%\right)$ (Fig. 3c).

The length of hospital stay Six studies [19-22, 27, 28] have reported the length of hospital stay, The summary WMDs on the length of hospital stay (days) was -0.30 (95\% CI: $-0.46--0.14)$, with evidence of heterogeneity $\left(P=0.28, \mathrm{I}^{2}=80 \%\right)$ (Fig. $\left.3 \mathrm{~d}\right)$.

The time to first bowel movement Two studies [27, 28] have reported the time to first bowel movement, The summary WMDs on the time to first bowel movement(hours) was $-5.38(95 \% \mathrm{CI}$ : $-7.39--3.38)$, with no evidence of heterogeneity $\left(P<0.001, \mathrm{I}^{2}=13 \%\right)$ (Fig. 3e).

Time to first feeling of hunger Three studies [21, 23, 28] have reported time to first feeling of hunger, The summary WMDs on time to first feeling of hunger (hours) was $-0.70(95 \% \mathrm{CI}:-2.97-1.57)$, with no evidence of heterogeneity $\left(P=0.32, \mathrm{I}^{2}=13 \%\right)$ (Fig. $\left.3 \mathrm{f}\right)$.

\section{Publication bias analysis}

Funnel plot, a simple scatter plot of the intervention effect estimates from every studies, is plotted against some measure of each study's size or precision (Cochrane Handbook for Systematic Reviews of Intervention. Part 2:8.5). Ten or more studies are required for the significant evidence of funnel plot. In our studies, due to the limitation of included data, we only performed funnel plot analysis on the time to first passage of flatus. As presented in Fig. 4, the distribution of study estimates in the funnel plot was symmetrical and well-proportioned, no significant publication bias have been found. 


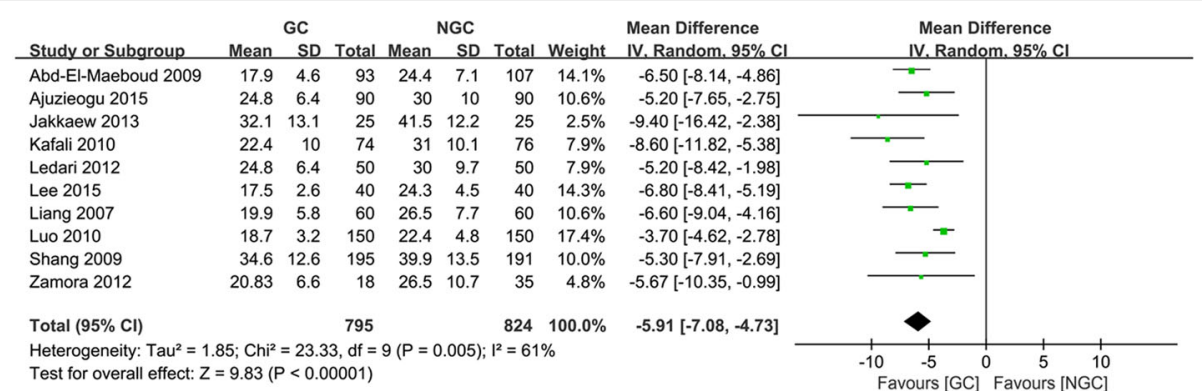

A The forest plot for time to first passage of flatus (h)

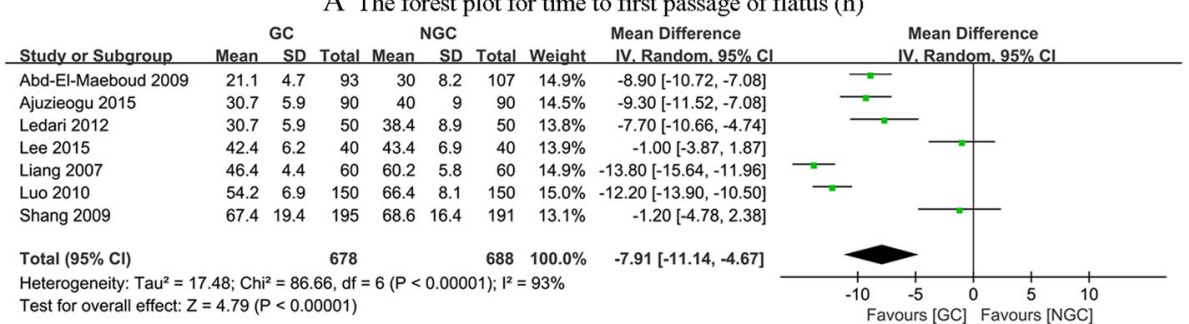

B The forest plot for time to first defecation(h)

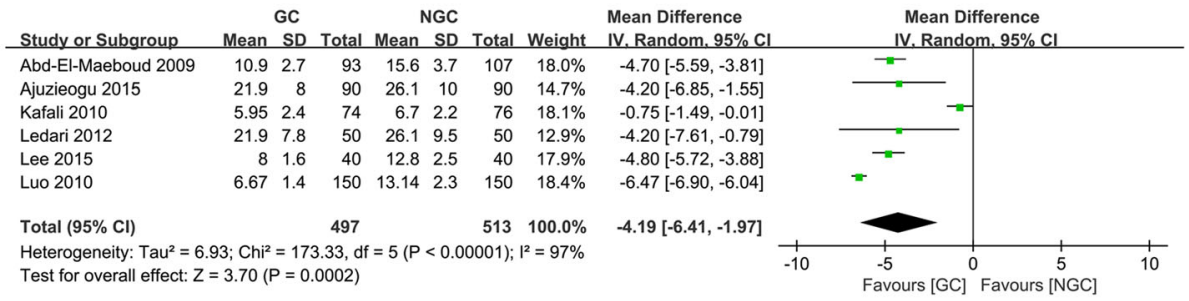

C The forest plot for time to first bowel sound(h)

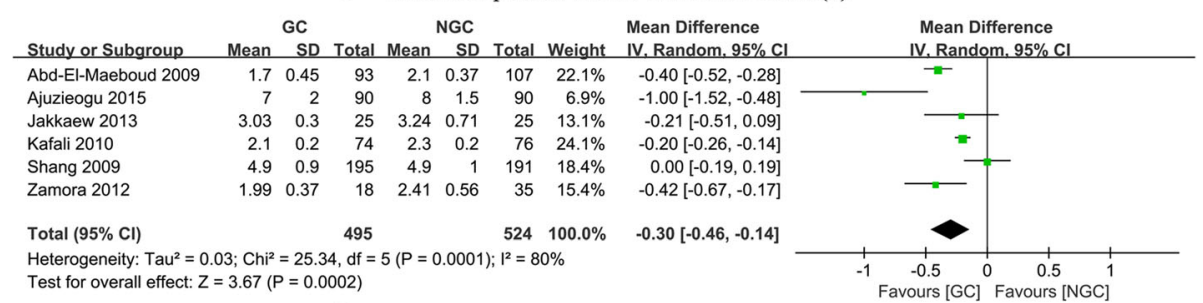

D The forest plot for length of hopital stay(d)

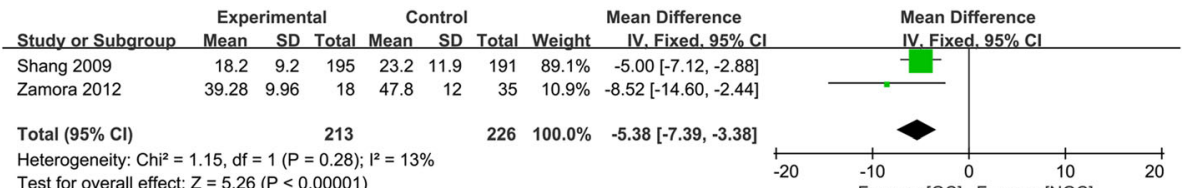

E The forest plot for time to first bowel movement(h)

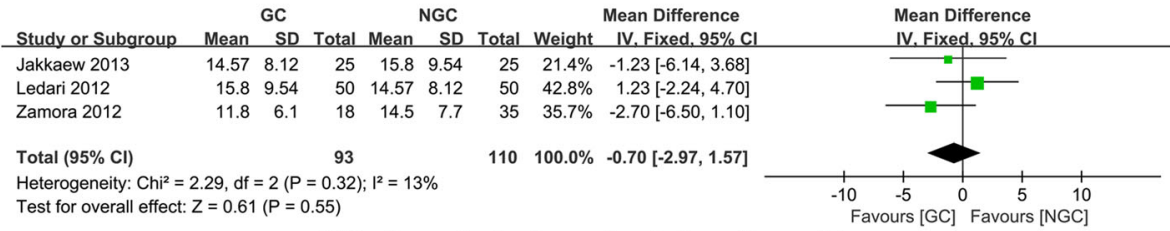

F The forest plot for time to first feeling of hunger(h)

Fig. 3 The forest plots for different outcomes

\section{Subgroup and sensitivity analyses}

No subgroup analyses were made in this study. Sensitivity analyses which investigate the impact of a single study on the overall risk estimate by abandoning one study in each turn. In the process of sensitivity analyses, we found that the results of time to first bowel movement changed when we neglected the study conducted by Shang [27], yet the data on this outcome still quite 


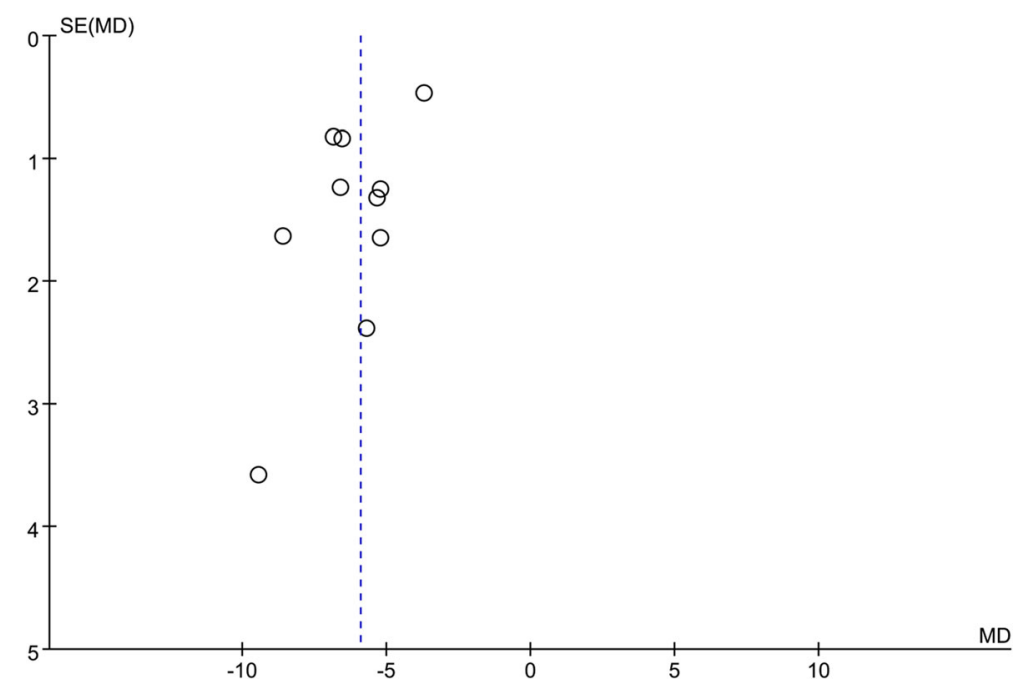

Fig. 4 The funnel plot for the time to first passage of flatus

limiting. For other outcomes, no significant change of the overall risk estimates by abandoning any single study was found.

\section{Discussion}

The results of our meta-analysis have indicated gum chewing after caesarean section can significantly promote the intestinal function recovery by accelerating the time to first passage of flatus, first defecation, first bowel sound, first bowel movement and shortening the length of hospital stay. The gum-chewing groups' faster recovery of gastrointestinal tract motility may result from stimulating intestinal motility by strengthening the cephalic-vagal reflex and increasing the gastrointestinal hormones secretion associated with bowel motility [9]. However, we did not find benefits of gum chewing on the time to first feeling of hunger, one possible explanation is that the chewing movement cause a feeding feedback to the brain, making a full feeling for our central nerve systems, additionally, the early feeding program employed in some studies can further enhance the hunger stimulus, previous study [10] has concluded that sham feeding confers no advantage if patients are placed on a rapid postoperative feeding regimen. Also, all the included studies have reported no side effects of gum chewing intervention in patients after caesarean section, therefore, gum chewing may be a safe and feasible intervention for accelerating the intestinal function recovery in patients after caesarean section.

Similar to other meta-analyses, our study has upheld the use of gum chewing in the early stage after caesarean section to accelerate the intestinal function recovery. A recent meta-analysis by Huang [12] included only five RCTs involving 882 patients, the sample size and number of included studies were small. An earlier meta- analysis by Hochner [15] also included five RCTs with a total of 846 participants, the process for meta-analysis was rather strict, yet the publication bias could not be evaluated quantitatively by a funnel plot due to the small number of studies available, the other meta-analyses [14, $16,17]$ also had problems in this area. Another comprehensive Cochrane meta-analysis [13] identified 81 studies involving 9072 participants to investigate whether the chewing gum after surgery hasten the return of gastrointestinal function after abdominal surgery, come to the finding that gum chewing showed a beneficial impact on the major outcomes of digestive system activation, including bowel sound, flatus passage and bowel movement, yet the including studies were rather heterogeneous. Different from previous meta-analysis, with more related RCTs published, our study has included more RCTs for synthesized analysis, which may have more advantages in reducing publication bias.

Several previous meta-analyses $[10,29]$ focused on the influence of chewing gum after colorectal cancer surgery, come the finding that it might promote the recovery of intestinal function. A meta-analysis [30] including12 RCTs focused on chewing gum and postoperative ileus in adults, found that chewing gum provided small benefit in reducing time to flatus, and time to bowel motion, but not in the length of stay or the incidence of complications, it's noteworthy that this studies included patients receiving either colorectal surgery or cesarean sections, in which lots of heterogeneities might exist, it should be emphasized that the surgery differences may lead to different injuries to the intestinal function, and gum chewing may offer different effects on the recovery process, specific intervention details(starting time, frequency et al.) of gum chewing for different surgeries remain further investigation. 
Although early oral intake has been promoted as standard care after caesarean section [31], only one RCT [22] adopted oral intake within six hours postpartum in both groups, still, when early oral intake was standardized in each group, the gum chewing added more benefits to the intestinal function recovery. A recent study [32] compared the effect of gum chewing, early oral hydration, and early mobilization on intestinal motility after cesarean birth, concluded that all of the three different interventions increased intestinal motility, and should be recommended during postoperative routine care to shorten hospital stay and prevent postoperative ileus, future research needs to further clarify the roles of those three interventions. Notably, one included RCT [24] also evaluated the effects of xylitol-containing and xylitol-free gum chewing on gastrointestinal recovery after cesarean section, the results indicated that xylitolcontaining gum might be superior to xylitol-free gum, while the most included RCTs adopted the sugar-free gum in their interventions. The sugar ingredients in sugar free gum (e.g., sorbitol and xylitol) may stimulate bowel mobility and exert a laxative effect, yet the evidence on whether sugar substitutes in sugar-free gum affect bowel function remain conflicting [33, 34], more researches in this area are also warranted.

Several limitations in this meta-analysis should be considered. First, there are some differences in gum chewing interventions (e.g., initiation, ingredients and frequency) among included RCTs, this is most likely accountable for the heterogeneity observed, however, due to the data limitations, we did not perform meta-regression analyses on patient population characteristics. Second, the quality of the included studies was not rather high, most included RCTs made no report on the randomization concealment and blinding, due to the nature of gum chew intervention, it's rather difficult to blind the participants in this study setting, but blinding the observers is achievable and might reduce the bias, future studies should put more attentions on the RCT design. Third, limited by study data, we did not perform subgroup analysis and funnel plot in some outcomes, still, it's possible that the end point susceptible to missing data from studies that were not published due to an overall null effect.

\section{Conclusions}

In conclusion, our study has further supported that gum chewing is associated with early recovery of intestinal function after caesarean section, which may be helpful to reduce the time to first passage of flatus, first defecation, first bowel sound, first bowel movement and shorten the length of hospital stay. Gum chewing offers a safe, simple and inexpensive for hastening the recovery of intestinal function after caesarean section, which is worthy of promotion for clinical use.

\section{Abbreviations}

CNKI: China National Knowledge Infrastructure; ORs: Odd ratios;

RCTs: Randomized controlled trials; WMDs: Weighted mean differences

\section{Acknowledgments}

None.

\section{Funding}

This research have received no specific grant from any funding agency in the public, commercial, or not-for-profit sectors.

\section{Availability of data and materials}

All data generated or analyzed during this study are included in this published article.

\section{Authors' contributions}

SMF and WZJ contributed to the conception and design of the research; WZJ, SMF DJP, MBB and WC contributed to the collection and analysis of the data; WZJ, SMF and WC contributed to the analysis and interpretation of the data; and WZJ wrote the first draft of manuscript. All authors have critically revised the manuscript, agreed to be fully accountable for ensuring the integrity and accuracy of the work, and read and approved the final manuscript.

\section{Competing interests}

The authors declare that they have no competing interests.

\section{Consent for publication}

Not applicable.

Ethics approval and consent to participate Not applicable.

\section{Publisher's Note}

Springer Nature remains neutral with regard to jurisdictional claims in published maps and institutional affiliations.

Received: 9 October 2016 Accepted: 21 March 2017

Published online: 18 April 2017

\section{References}

1. Degani N, Sikich N. Caesarean delivery rate review: an evidence-based analysis. Ont Health Technol Assess Ser. 2015;15(9):1-58.

2. Whitehead WE, Bradley CS, Brown MB, Brubaker L, Gutman RE, Varner RE, Visco AG, Weber AM, Zyczynski H, Pelvic Floor N. Gastrointestinal complications following abdominal sacrocolpopexy for advanced pelvic organ prolapse. Am J Obstet Gynecol. 2007;197(1):78 e71-77.

3. Allen AM, Antosh DD, Grimes CL, Crisp CC, Smith AL, Friedman S, McFadden BL, Gutman RE, Rogers RG. Management of ileus and smallbowel obstruction following benign gynecologic surgery. Int J Gynaecol Obstet. 2013;121(1):56-9.

4. Doorly MG, Senagore AJ. Pathogenesis and clinical and economic consequences of postoperative ileus. Surg Clin North Am. 2012;92(2):259-72. viii.

5. Stewart D, Waxman K. Management of postoperative ileus. Dis Mon. 2010;56(4):204-14.

6. Selby LV, Rifkin MB, Yoon SS, Ariyan CE, Strong VE. Decreased length of stay and earlier oral feeding associated with standardized postoperative clinical care for total gastrectomies at a cancer center. Surgery. 2016; 160(3):607-12.

7. Aydin Y, Altunyurt S, Oge T, Sahin F. Early versus delayed oral feeding after cesarean delivery under different anesthetic methods-a randomized controlled trial anesthesia, feeding in cesarean delivery. Ginekol Pol. 2014:85(11):815-22.

8. Hasler WL. Pharmacotherapy for intestinal motor and sensory disorders. Gastroenterol Clin North Am. 2003;32(2):707-32. viii-ix.

9. Lunding JA, Nordstrom LM, Haukelid AO, Gilja OH, Berstad A, Hausken T. Vagal activation by sham feeding improves gastric motility in functional dyspepsia. Neurogastroenterol Motil. 2008;20(6):618-24

10. Ho YM, Smith SR, Pockney P, Lim P, Attia J. A meta-analysis on the effect of sham feeding following colectomy: should gum chewing be included in enhanced recovery after surgery protocols? Dis Colon Rectum. 2014;57(1):115-26. 
11. Su'a BU, Pollock TT, Lemanu DP, MacCormick AD, Connolly AB, Hill AG. Chewing gum and postoperative ileus in adults: a systematic literature review and meta-analysis. Int J Surg. 2015;14:49-55.

12. Huang HP, He M. Usefulness of chewing gum for recovering intestinal function after cesarean delivery: A systematic review and meta-analysis of randomized controlled trials. Taiwan J Obstet Gynecol. 2015;54(2):116-21.

13. Short V, Herbert G, Perry R, Atkinson C, Ness AR, Penfold C, Thomas S, Andersen HK, Lewis SJ. Chewing gum for postoperative recovery of gastrointestinal function. Cochrane Database Syst Rev. 2015;2:CD006506.

14. Zhu YP, Wang WJ, Zhang SL, Dai B, Ye DW. Effects of gum chewing on postoperative bowel motility after caesarean section: a meta-analysis of randomised controlled trials. BJOG. 2014;121(7):787-92.

15. Hochner H, Tenfelde SM, Abu Ahmad W, Liebergall-Wischnitzer M. Gum chewing and gastrointestinal function following caesarean delivery: a systematic review and meta-analysis. J Clin Nurs. 2015;24(13-14):1795-804.

16. Craciunas L, Sajid MS, Ahmed AS. Chewing gum in preventing postoperative ileus in women undergoing caesarean section: a systematic review and meta-analysis of randomised controlled trials. BJOG. 2014;121(7):793-9. discussion 799.

17. Yuan $Y, Z$ Zhao $H, H$ J, Gong $X$. Chewing gum in promoting bowel recovery after cesarean section: a systematic review. Chin J Evid Based Med. 2011;11(4):6.

18. Liberati A, Altman DG, Tetzlaff J, Mulrow C, Gotzsche PC, loannidis JP, Clarke M, Devereaux PJ, Kleijnen J, Moher D. The PRISMA statement for reporting systematic reviews and meta-analyses of studies that evaluate health care interventions: explanation and elaboration. PLoS Med. 2009;6(7):e1000100.

19. Abd-El-Maeboud KH, Ibrahim MI, Shalaby DA, Fikry MF. Gum chewing stimulates early return of bowel motility after caesarean section. BJOG. 2009;116(10):1334-9.

20. Ajuzieogu OV, Amucheazi A, Ezike HA, Achi J, Abam DS. The efficacy of chewing gum on postoperative ileus following cesarean section in Enugu, South East Nigeria: A randomized controlled clinical trial. Niger J Clin Pract. 2014;17(6):739-42.

21. Jakkaew B, Charoenkwan K. Effects of gum chewing on recovery of bowel function following cesarean section: a randomized controlled trial. Arch Gynecol Obstet. 2013;288(2):255-60.

22. Kafali H, Duvan Cl, Gozdemir E, Simavli S, Onaran Y, Keskin E. Influence of gum chewing on postoperative bowel activity after cesarean section. Gynecol Obstet Invest. 2010;69(2):84-7.

23. Ledari FM, Barat S, Delavar MA. Chewing gums has stimulatory effects on bowel function in patients undergoing cesarean section: a randomized controlled trial. Bosn J Basic Med Sci. 2012;12(4):265-8.

24. Lee JT, Hsieh MH, Cheng PJ, Lin JR. The role of xylitol gum chewing in restoring postoperative bowel activity after cesarean section. Biol Res Nurs. 2016:18(2):167-72.

25. Liang J, Gao Z, Han W, Zhang G, Liu S, Dai Q. The clinical observation of enhancing recovery of gastrointestinal function after cesarean section by gum chewing. J Tongji Univ (Med Sci). 2007;28(2):3.

26. Luo S, Wu C, Yang X, Lei L, Deng H, Li H. Effect of chewing gum afar cesarean section Oll restoration of gastrointestinal function. J Mod Nurs. 2010;16(24):2.

27. Shang $H$, Yang Y, Tong $X$, Zhang L, Fang A, Hong L. Gum chewing slightly enhances early recovery from postoperative ileus after cesarean section: results of a prospective, randomized, controlled trial. Am J Perinatol. 2010;27(5):387-91.

28. Zamora BBB, Kalalo RE. Abstracts of XX FIGO World congress of gynecology and obstetricsO752 GUM chewing versus traditional feeding on the early return of bowel motility after cesarean delivery: a prospective randomized controlled trial. Int J Gynecol Obstet. 2012;119:S525.

29. Purkayastha S, Tilney HS, Darzi AW, Tekkis PP. Meta-analysis of randomized studies evaluating chewing gum to enhance postoperative recovery following colectomy. Arch Surg. 2008;143(8):788-93.

30. Noble EJ, Harris R, Hosie KB, Thomas S, Lewis SJ. Gum chewing reduces postoperative ileus? a systematic review and meta-analysis. Int I Surg. 2009; 7(2):100-5.

31. Millen KR, Kuo K, Zhao L, Gecsi K. Evidence-based guidelines in labor management. Obstet Gynecol Surv. 2014;69(4):209-17.

32. Sahin E, Terzioglu F. The effect of gum chewing, early oral hydration, and early mobilization on intestinal motility after cesarean birth. Worldviews Evid Based Nurs. 2015;12(6):380-8.

33. Storey D, Lee A, Bornet F, Brouns F. Gastrointestinal tolerance of erythritol and xylitol ingested in a liquid. Eur J Clin Nutr. 2007;61(3):349-54.

34. Tandeter H. Hypothesis: hexitols in chewing gum may play a role in reducing postoperative ileus. Med Hypotheses. 2009;72(1):39-40.

\section{Submit your next manuscript to BioMed Central and we will help you at every step:}

- We accept pre-submission inquiries

- Our selector tool helps you to find the most relevant journal

- We provide round the clock customer support

- Convenient online submission

- Thorough peer review

- Inclusion in PubMed and all major indexing services

- Maximum visibility for your research

Submit your manuscript at www.biomedcentral.com/submit
Biomed Central 